\title{
Calibration and Monitoring of the Tile Calorimeter during LHC Run-I
}

\author{
Smita DARMORA* \\ On behalf of the ATLAS Tile Calorimeter System \\ University of Texas at Arlington (US) \\ E-mail: smita.darmora@cern.ch
}

The ATLAS hadronic calorimeter, named the Tile Calorimeter (TileCal), is a non-compensating sampling calorimeter comprised of steel and scintillating plastic tiles which are read-out by photomultiplier tubes (PMTs). The TileCal is regularly monitored and calibrated by several different calibration systems: a Cs radioactive source that illuminates the scintillating tiles directly, a laser light system to directly test the PMT response, and a charge injection system (CIS) for the frontend electronics. These calibrations systems, in conjunction with data collected during protonproton collisions, provide extensive monitoring of the instrument and a means for equalizing the calorimeter response at each stage of signal propagation. Through the individual calibrations provided by these systems, we are able to achieve a precision of approximately $0.5-1.0 \%$ in the monitoring of the evolution of the response of the different components of TileCal. Analysis of the combined calibrations is used to measure the gain variance in the detector response. This contribution presents a brief description of the different TileCal calibration systems along with the latest results on their performance during the LHC Run I.

Technology and Instrumentation in Particle Physics 2014,

2-6 June, 2014

Amsterdam, the Netherlands

\footnotetext{
*Speaker.
} 


\section{The Tile Calorimeter}

The ATLAS Tile Calorimeter (TileCal) is the hadronic calorimeter covering the central region $|\eta|<1.7$ [1]. It contributes to the measurement of the missing transverse energy, hadrons, taus and jets. TileCal is a non-compensating sampling calorimeter, composed of alternating layers of scintillating tiles and steel plates. TileCal is broken up into three sections: one long barrel and two extended barrels labeled as EBA and EBC shown in Fig.1 (left). The long barrel is split into two readout partitions LBA and LBC. Each barrel section of TileCal is composed of 64 modules, which is composed of three longitudinal layers (A, BC and D). Hadronic showers developing in the TileCal create light in the scintillating tiles, which is transmitted by wavelength shifting fibers and readout by photomultiplier tubes (PMTs). The signal collected by each PMT is digitized and transmitted to the TileCal readout system and is shown in Fig.1 (right). The calibration systems described in the following section are designed to monitor all the readout systems: the optical properties of the scintillators, the PMT gains, and the front-end electronics.
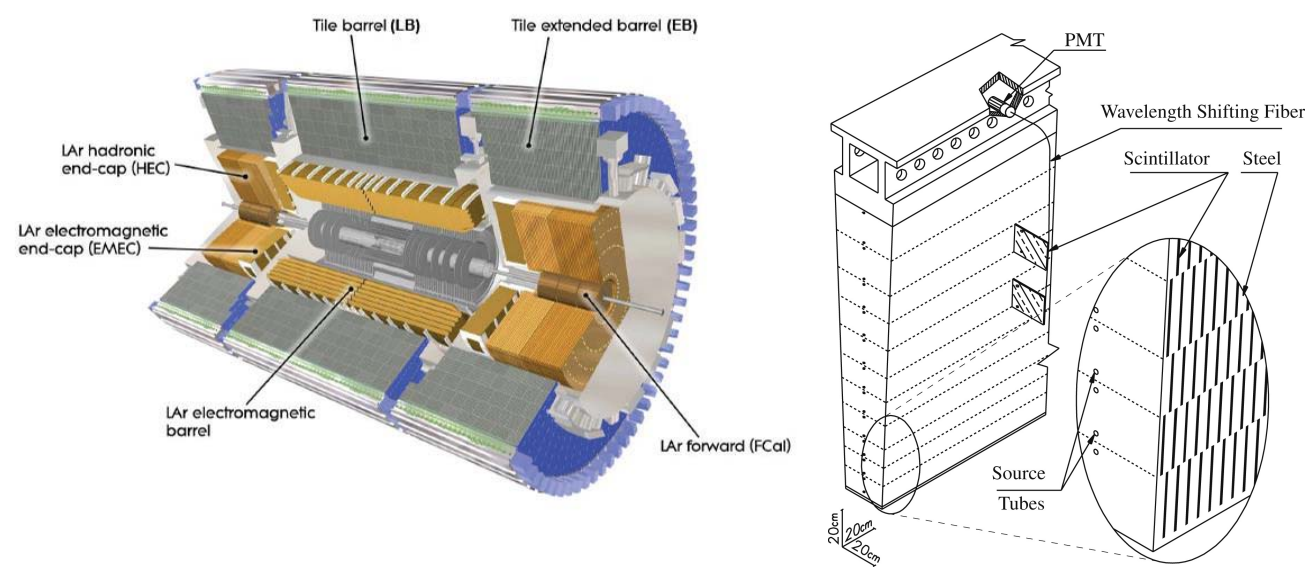

Figure 1: Tile Calorimeter system of the ATLAS detector (left) and the structure of a module of the Tile Calorimeter, showing alternating steel and scintillating materials (right).

\section{The Energy Calibration systems in TileCal}

The TileCal calibration relies on several dedicated systems to monitor and equalize the calorimeter response at each stage of signal generation: Cesium, Laser and The Charge Injection System (CIS). Calibration systems adjust the energy on a channel-by-channel basis and identify potential hardware failures. For each channel, the calibrated energy is calculated as:

$$
E(\mathrm{GeV})=A_{\text {opt }}[\mathrm{GeV}] \cdot C_{C I S} \cdot C_{l} \cdot C_{C s} \cdot C_{e}
$$

Where $A_{o p t}$ is the amplitude in ADC counts, $C_{C I S}$ is the $A D C \rightarrow p C$ conversion factor as determined by the CIS system, $C_{l} \& C_{C s}$ are relative correction factors measured by the cesium and laser system, and $C_{e}$ is the $p C \rightarrow G e V$ conversion factor derived from the response to electrons in past beam tests. 


\subsection{Calibration Systems of TileCal}

The cesium system is the primary tool to determine the electromagnetic (EM) scale and equalize the response of the TileCal cells [2]. It is based on three movable radioactive ${ }^{137} \mathrm{Cs}$ sources using hydraulic control. Fig.2 (left) shows the evolution of the response to the cesium source as a function of time in the four partitions of the TileCal. Fig.2 (right) shows the deviation of the measured cesium response from expected response, relative to the response measured in June 2009 without magnetic field, therefore showing an increase of response in the presence of magnetic field.
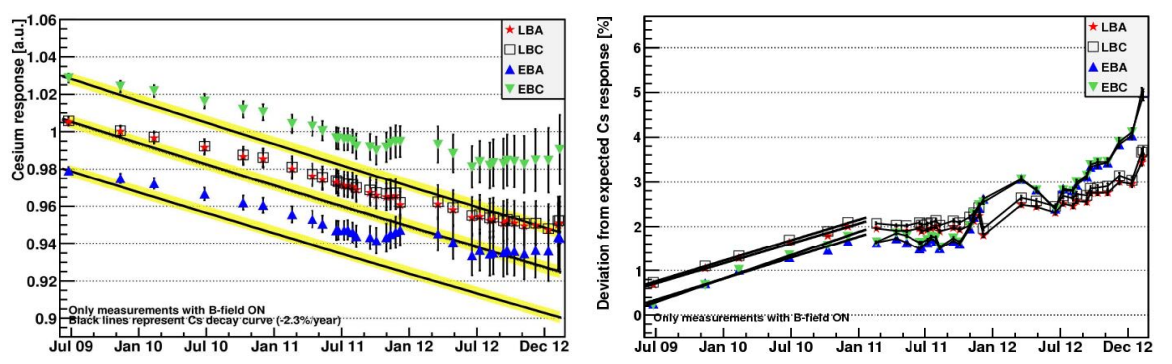

Figure 2: The left plot shows evolution of the average TileCal response to cesium source as a function of time. Three families of points correspond to three different cesium sources used for calibration. The black line represents the cesium decay curve. The right plot shows deviation of measured signal from expected values, if the response would follow the cesium decay curve.

The laser calibration system is dedicated to the monitoring and calibration of the gain and the linearity of each TileCal PMT [3]. A calibrated laser light is sent to the PMTs and the low and high gain variation is extracted by reconstructing the signal. Deviation of any channel response with respect to its nominal response gives the calibration constant for the laser system. The precision of the laser system is sufficient to observe average PMT gain variation of $<2 \%$, which mostly affects the cells at the inner radius that have a higher current, as can be seen in Fig.3 (left).

The Charge Injection System (CIS) calibrates the gain of the front-end electronics and measures the ADC counts/pC factor relating pulse amplitude to electric charge. It provides a quantitative relation between the analog signal from TileCal PMTs and electronics response of TileCal read-out channels and corrects the non-linearity in the signal. The CIS is generally very stable with a precision of $0.7 \%$ (Fig.3 (right) [4]).

\section{Combined Calibration and Calibration of Gap and Crack region}

Combining results from all the calibrations system can determine possible sources drift (see in Fig.4). Average up-drift of about $0.8 \%$ was observed during 2009-2010, but the effect disappeared in 2011 when the LHC started operating at higher luminosity and sizable down-drift is seen. After the 2010 technical stop, a global tendency toward up-drift is observed, with some oscillation. During data-taking operations, we observe a reduced response, but this recovers during shutdown periods [4].

The Intermediate Tile Calorimeter (ITC) is a structural extension of the TileCal in the gap region. The gap scintillators, E1 and E2 cells are monitored by the laser and cesium but are cal- 

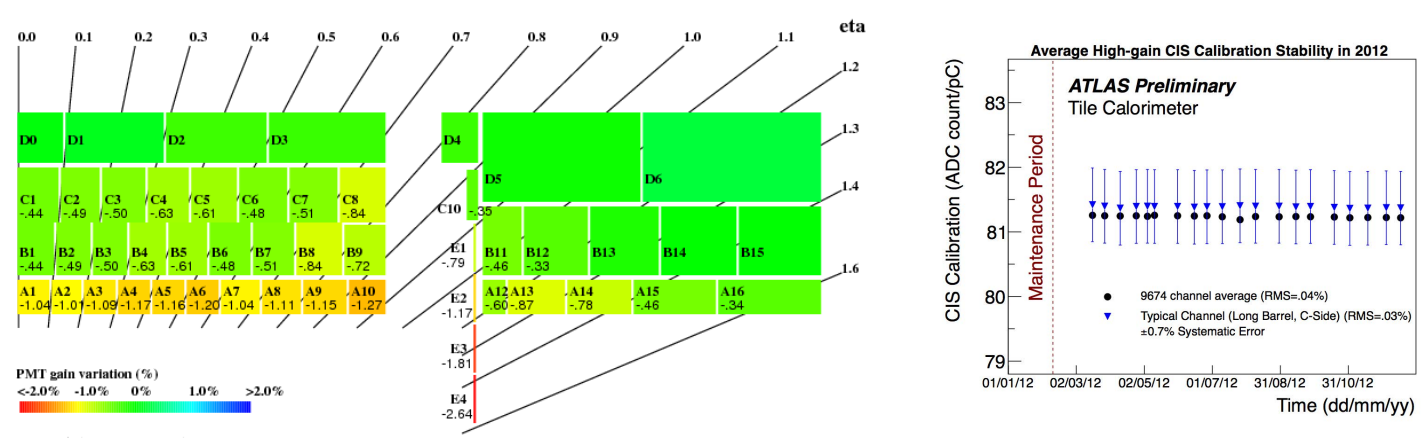

Figure 3: (left) Average PMT gain variation (in \%) in the TileCal cells between two consecutive cesium scans. (right) Time stability of the average high-gain read-out calibration constants from Feburary 2012 to December 2012.
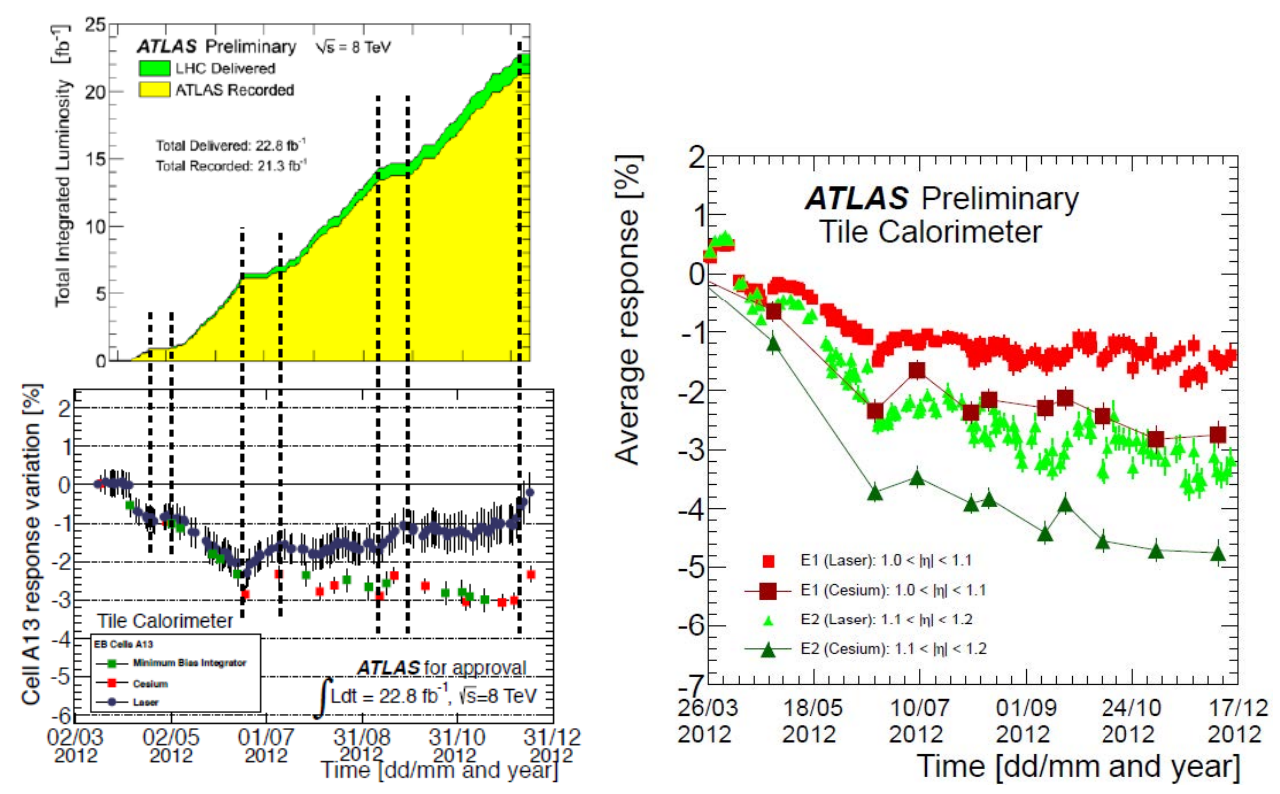

Figure 4: Gain variation measured by cesium and laser systems, averaged over A13 cell type, compared to LHC integrated luminosity (left). Right plot shows the similar behavior of E1 and E2 cells to the rest of the TileCal.

ibrated only with the cesium system. A dedicated cesium method is used to calibrate the ITC, which shows a similar behavior to the rest of the TileCal in terms of down-drift and up-drift of the response. Fig.4 (right) shows the maximal drift of $-3 \%$ observed by the laser system in 2012 .

\section{Conclusion}

The combined calibration system results allow for very efficient monitoring and prompt corrections of the TileCal. These calibrations have shown to be stable over time. A cesium calibration run takes place every month. In between cesium runs, the PMT response is calibrated via the laser 
system. Both systems are in excellent agreement for non-ITC cells. The stability of the absolute energy scale at the cell level was maintained at a level better than $1 \%$ during the LHC Run-I.

\section{References}

[1] ATLAS Collaboration, Readiness of the ATLAS Tile Calorimeter for LHC collisions.The European Physical Journal C 70 1193-1236, 2010

[2] E.A. Starchenko et al., Cesium monitoring system for ATLAS Tile Hadronic Calorimeter. Nucl. Instrum. Meth. A 494 (2002) 381.

[3] V. Giangiobbe on behalf of the ATLAS Tile Calorimeter System, The TileCal Laser calibration system. ATL-TILECAL-PROC-2011-007,CERN (https://cds.cern.ch/record/1381507)

[4] ATLAS Collaboration, Public Tile Calorimeter Plots for Collision Data. https://twiki.cern.ch/twiki/bin/view/AtlasPublic/ApprovedPlotsTileCalibration 